\title{
VAC_07 - CLEC5A expression on monocytes may be a good marker to characterize early immunity signature after yellow fever immunization
}

Juliana Gil Melgaço ${ }^{1 *}$; Tamiris Azamor da Costa Barros ${ }^{1}$; Andréa Marques Vieira da Silva ${ }^{1}$; Luciana Neves Tubarão ${ }^{1}$; Ana Paula dos Santos ${ }^{1}$; Denise Cristina de Souza Matos ${ }^{1}$; Ana Paula Dinis Ano Bom ${ }^{1}$; Milton Ozório Moraes ${ }^{2}$; Patrícia Cristina da Costa Neves ${ }^{1}$.

${ }^{1}$ Fiocruz/Bio-Manguinhos;

${ }^{2}$ Fiocruz/IOC.

Introduction: It has been known that Yellow Fever (YF) vaccine is one of the most effective ever made with more than 540 million doses administered globally. Vaccination with YF17D virus induces a strong $\mathrm{T}$ CD8 + responses and high titers of neutralizing antibodies. Even though YF vaccine has been associated with rare cases of serious adverse events, immunological mechanism involved are not well understood. In addition, there are few studies about how innate immune events may have a role in the good response after vaccination. A transmembrane C-leptin type receptor CLEC5A in mononuclear cells has been associated to poor outcomes on other flavivirus infections.

Objective: In this concept, this study was aimed to investigate the role of CLEC5A after YF vaccination.

Methodology: Here, 34 subjects were followed pre and after YF vaccination using the Brazilian vaccine (17DD), with blood sample collection from $0,3,4,5,7,10$, and 30 days after immunization (DAI). Blood samples were used to perform quantification of YF antibodies, and in vitro antigen stimulation for immunophenotyping and gene expression assays. The peripheral blood mononuclear cells (PBMC) were isolated by Ficoll-Paque ${ }^{\circledR}$ gradient and submitted to FACS assay to investigate classical monocytes expressing CLEC5A on 0, 3, 5 and 7 DAI. PBMC were also used for RNA extraction using TRIzol ${ }^{\circledR}$, followed by cDNA synthesis with High-Capacity cDNA Reverse Transcription kit. Quantitative Polymerase Chain Reaction (qPCR) assay was carried out in samples from 0, 4, 7 and 10 DAI, using SYBR green ${ }^{\circledR}$ to quantify expression of CLEC5A. Serum was collect 30 DAI and used to perform Virus Binding inhibition assay (Vibi) to quantify antibodies against yellow fever.

Results: Our findings showed that all subject presented YF antibodies 30 days after immunization. After in vitro YF virus (YF17DD) stimulation, it was observed that a subpopulation of activated monocytes (CD3CD95-CD14+HLADR+) expressing CLEC5A had their frequency significantly increased on the fifth day after immunization and it was correlated with antibodies production $(\mathrm{p}=0.002)$, although there were not significant changes noted in the frequency of monocytes activated (CD3-CD95-CD14+HLADR+) along follow-up ( $\mathrm{p}=0.36$ ). For mRNA relative expression analysis, CLEC5A gene was highly expressed in samples collected 4-5 days after immunization. There is a slightly positive correlation between CLEC5A gene expression and YFV-specific activated monocytes expressing CLEC5A until 5 days after immunization, therefore it was not significant (Spearman $\mathrm{R}=0.34, \mathrm{p}=0.19$ ).

Conclusion: These results indicated that monocytes expressing CLEC5A might be a signature for early immunity after vaccination. In addition, findings showed that cellular immune response was related to antibodies production, and consequently, combined with gene expression those markers may be useful in the future to predict protection after YF immunization.

Keywords: yellow fever vaccine; innate immunity; CLEC5A 